\title{
Left Ventricular Noncompaction Combined With Epinephrine- Secreted Pheochromocytoma Inducing Heart Failure
}

\author{
A Case Report
}

\author{
Ling Han, ${ }^{1}$ MD, Jing-Gang Luo, ${ }^{1} \mathrm{MD}, \mathrm{Xin}$ CHen, ${ }^{1} \mathrm{MD}$, Wen-Ze Hu, ${ }^{1} \mathrm{MD}$, \\ Li-Wei Chen, ${ }^{1}$ MD, Xiao-Ming XIN, ${ }^{1}$ MD, Ming Yang, ${ }^{1}$ MD, Jun DuAn,,${ }^{1}$ MD, \\ Feng-Jun Zou, ${ }^{1} \mathrm{MD}, \mathrm{Xu}$ Teng, ${ }^{2} \mathrm{MD}$, and Yong-Fen QI, ${ }^{3} \mathrm{MD}$
}

\begin{abstract}
SUMMARY
Pheochromocytomas and left ventricular noncompaction (LVNC) are both rare diseases. In this patient, the long duration of the catecholamine-secreted pheochromocytoma caused myocardial ischemia, pressure overload, and hypertrophy, resulting in the onset of heart failure (HF). The LVNC might be associated with the acute attack of HF induced by the pheochromocytoma. This is the first case reporting LVNC in combination with HF secondary to pheochromocytoma. (Int Heart J 2016; 57: 254-257)
\end{abstract}

Key words: Hypertension, Myocardial remodeling, Catecholamines, Human

$\mathrm{P}$ heochromocytomas are rare neuroendocrine tumours that originate in chromaffin tissue and produce their distant variant effects by secretion of catecholamines that tend to mislead physicians into making a wrong diagnosis. ${ }^{1)}$ Although they have only about a $5 \%$ incidence of malignancy, these tumors are associated with a high risk of morbidity and mortality from cardiovascular complications. ${ }^{2)}$ Cardiovascular complications of pheochromocytomas present as heart failure (HF), hypertension, cardiac hypertrophy, arrhythmias, or myocardial infarction, although many patients are asymptomatic and the condition may be incidentally diagnosed on imaging modalities. ${ }^{3-5)}$

In 1932, the first case of noncompaction was reported after an autopsy performed on a newborn infant with an aortic atresia/coronary-ventricular fistula. In 1984, the first isolated noncompaction cardiomyopathy was described. ${ }^{6}$ Left ventricular noncompaction (LVNC), also known as "spongy myocardium", is a rare abnormality of the left ventricular myocardium with two layers: a compacted layer and a noncompacted layer. ${ }^{7)}$ LVNC was first reported in children and has recently become increasingly diagnosed in adults, which is associated with high morbidity and mortality. ${ }^{8)}$ The clinical picture of LVNC patients ranges from no symptoms to HF, thromboembolic events, arrhythmias, and sudden death

Making a correct diagnosis of LVNC is important as it can significantly influence decisions on long-term management and prognosis. A correct diagnosis of LVNC has impor- tant implications due to the possible association with other cardiac abnormalities, neuromuscular disorders, and/or other systemic anomalies. Here, we report an unusual case of pheochromocytoma causing LVNC with HF.

\section{Case Report}

A 28-year-old female patient presented to our Coronary Care Unit (CCU) department with "palpitations and shortness of breath for 4 days" on August 12, 2010. Paroxysmal episodes of headache were noted every 1 to 2 months for 8 years, occasionally with sweating and palpitations. No other symptoms or signs including fever, drug or alcohol abuse, or smoking were reported. Her family history was unremarkable. On physical examination, her pulse was 110 beats per minute and her blood pressure was 130/100 mmHg. Cardiovascular examination revealed an expanding left heart border and tachycardia. Mild edema of both lower limbs was seen. Few bibasilar rales were auscultated in the lungs. Her physical examination suggested biventricular heart failure. Electrocardiography (ECG) showed sinus tachycardia with ST-segment depressions by leads I, aVL, and V1-V6. Myocardial enzymes were significantly increased (Table I). Transthoracic echocardiography showed cardiac hypertrophy and dilatation, global hypokinesia with low left ventricular ejection fraction (LVEF), left atrial dilatation, and papillary muscle thickening with slight mitral regurgitation

From the ${ }^{1}$ Department of Cardiology, Fu Xing Hospital, Capital Medical University, Beijing, ${ }^{2}$ Hebei Key Laboratory of Laboratory Animal Science, Hebei Medical University, Shijiazhuang, ${ }^{3}$ Key Laboratory of Molecular Cardiovascular Science, Ministry of Education, Peking University Health Science Center, Beijing, China.

This work was supported by the National Natural Science Foundation of China. (No. 9139203)

Address for correspondence: Yong- Fen Qi, MD, Key Laboratory of Molecular Cardiovascular Science, Ministry of Education, Peking University Health Science Center, Xueyuan Road No.38, Haidian District, Beijing 100191, China. E-mail: yongfenqi@ 163.com or Xu Teng, MD, Hebei Key Laboratory of Laboratory Animal Science, Hebei Medical University, Zhongshan East Road No. 361, Changan District, Shijiazhuang, Hebei 050017, China. E-mail: tengxu78@163.com

Received for publication August 9, 2015. Revised and accepted September 27, 2015.

Released in advance online on J-STAGE March 11, 2016.

All rights reserved by the International Heart Journal Association. 
Table I. Serum Levels of Myocardial Enzymes Before and After Treatment

\begin{tabular}{lcc} 
& Before treatment & After treatment \\
\hline Creatine kinase (CK, IU/L) & 120 & 62 \\
MB isoenzyme of creatine kinase (CK-MB, IU/L) & 50.6 & 30 \\
Troponin T (ng/mL) & 0.092 & 0.014 \\
Brain natriuretic peptide (BNP, pg/mL) & 532 & 274 \\
\hline
\end{tabular}

Normal levels: CK, 1-120 IU/L; CK-MB, 0-24 IU/L; Troponin T, 0-0.014 ng/mL; BNP, 0-100 pg/mL.

Table II. Echocardiographic Parameters Before and After Treatment

\begin{tabular}{lll}
\hline & Before treatment & After treatment \\
\hline Anteroposterior diameter of left atrium (mm) & 43 & 36 \\
Left ventricular diastolic diameter (LVIDd, mm) & 57.3 & 47.1 \\
Thickness of left ventricular in the apex (mm) & 23 & 21 \\
Left ventricular ejection fraction (LVEF, $\%)$ & 27.2 & 56 \\
\hline
\end{tabular}

(Table II). Myocarditis and acute left heart failure were suspected on admission.

Following HF treatment with bed rest, sodium nitroprusside and diuretics, the patient still experienced episodes of hypertension $(200 / 120 \mathrm{mmHg})$ with palpitations, headaches, sweating, and nausea. Thoracic-abdominal computed tomography $(\mathrm{CT})$ images showed a solid hypotense mass $(3.7 \mathrm{~cm} \times 3$ $\mathrm{cm})$ in the right adrenal gland with relatively well-defined borders. Given the suspicion of pheochromocytoma, 24 hour urinary excretions of total metanephrines and catecholamines were measured, which showed elevation of epinephrine in urine specimens. After treatment with the $\alpha / \beta$-adrenergic receptor antagonist carvedilol (20 mg bid), the blood pressure was controlled $(120 / 70 \mathrm{mmHg})$ and the symptoms of the patient were relieved. After 12 days of treatment in hospital, the serum levels of myocardial enzymes were all decreased (Table I). Two weeks later, echocardiography showed all previous symptoms had improved (Table II). However, the thickened left ventricle had a double-wall structure. In the lateral wall, the compacted layer thickness was about $5 \mathrm{~mm}$, and the noncompaction layer was $16.6 \mathrm{~mm}$ at diastole. A number of excessively prominent trabeculations and deep intertrabecular recesses in the apical segments of the left ventricle were seen. Doppler diagnosis indicated that blood flow in the cardiac chambers was connected with recesses (Figure 1). Cardiac magnetic resonance (CMR) showed the left ventricular end diastolic diameter was $60 \mathrm{~mm} \times 87 \mathrm{~mm}$, left ventricular systolic and diastolic movement was weakened, and much trabecular muscle was seen inside the wall of the left ventricle (Figure 2). These results confirmed the diagnosis of LVNC. The patient was discharged from hospital with amelioration of symptoms after continued diuretic and blood pressure control.

After two months, the 28-year-old patient was re-hospitalized, and resection of the pheochromocytoma was performed. All oral drugs were ceased after the operation. Until the patient was 29 years old, she had no episodes of palpitations, dyspnea, tachycardia, or hypertension. Echocardiography was performed again at 30 years old, and the results still clearly showed a compacted layer of about $5 \mathrm{~mm}$, a non-compaction layer of about $16.6 \mathrm{~mm}$, ventricular trabeculations and deep intertrabecular recesses in the apex and lateral wall, and blood flow running through the recesses and cardiac chambers.

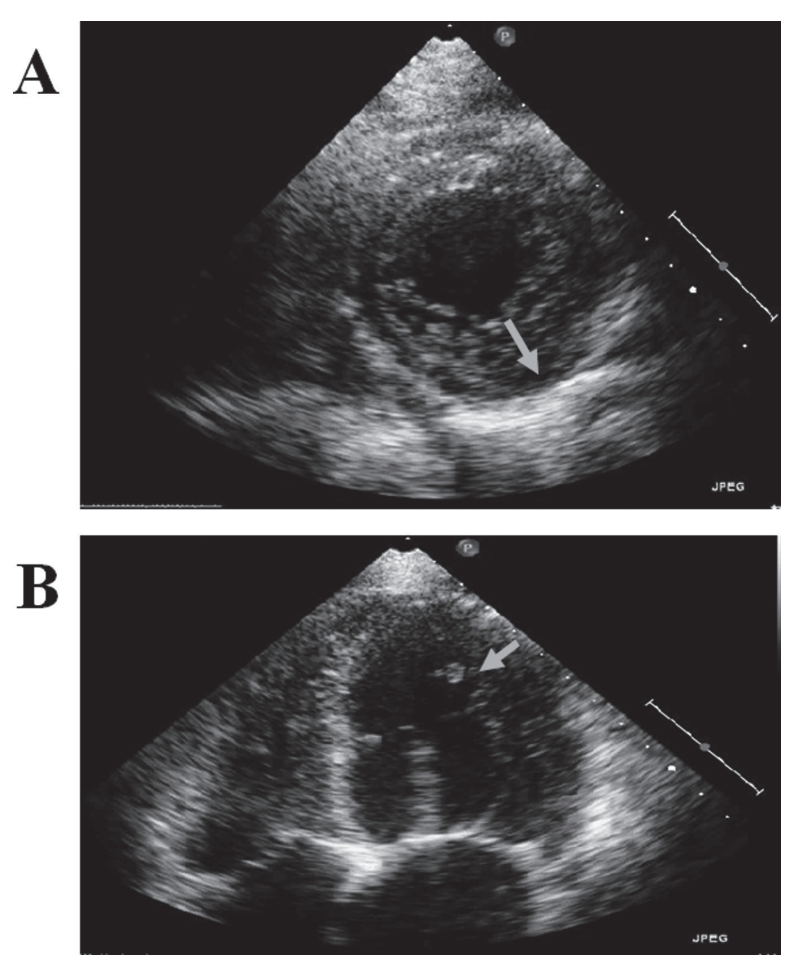

Figure 1. Echocardiogram obtained on August 13, 2010 showed the left ventricular wall was thickened, especially in the apex. The non-compaction layer was about $16.6 \mathrm{~mm}$, while the compacted layer was $5 \mathrm{~mm}$. Ventricular trabeculations and deep intertrabecular recesses were obvious in the apex and lateral wall, and the blood flow ran through the recesses and chambers of the heart. A: short-axis view of the heart, and B: 4-chamber view.

However, the systolic function of the myocardium was significantly improved, and LVEF was increased to 56\% (Figure 3).

\section{Discussion}

LVNM is a rare cardiomyopathy characterized by hypertrabeculation of the myocardium, ${ }^{9}$ and it is related to a high 


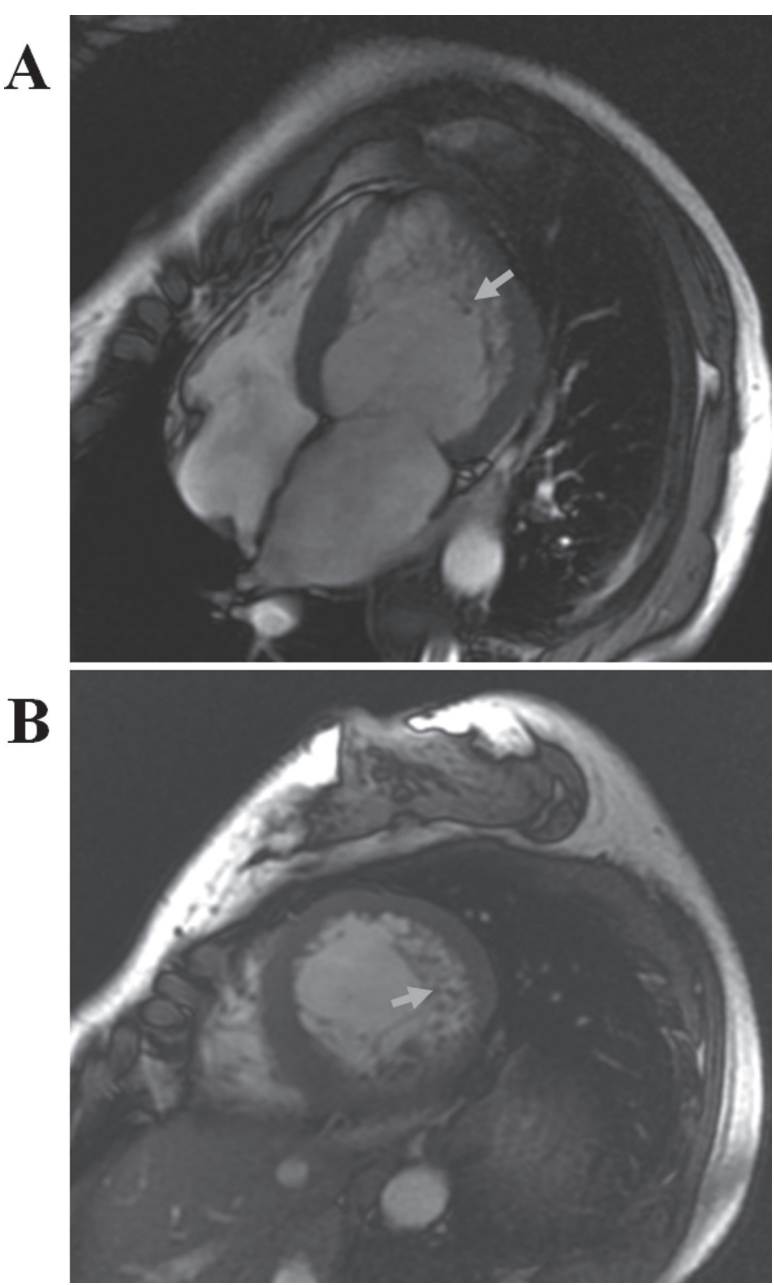

Figure 2. Cardiac magnetic resonance imaging showed much trabecular muscle inside the wall of the left ventricular. A: long-axis view of the heart, and B: 4-chamber view.

incidence of death or heart transplantation. ${ }^{10,11)}$ However, the diagnosis of LVNC was usually missed because its clinical picture is nonspecific. Routine transthoracic echocardiography is an excellent tool with which to diagnose LVNC. ${ }^{12)}$ Currently, 3 non-standardized sets of criteria to diagnose LVNC by echocardiography exist. ${ }^{13-15)}$ The characteristic echocardiographic findings of noncompaction are multiple, prominent myocardial trabeculations and deep intertrabecular recesses communicating with the LV cavity. Color Doppler flow imaging shows blood flow through these deep recesses in continuity with the ventricular cavity. Also, a two-layered structure between the noncompacted layer and the compacted layer is consistent with LVNC. ${ }^{16)}$ In this case, the echocardiographic imaging showed ventricular trabeculations and deep intertrabecular recesses in the apex and lateral wall, and blood flow running through the recesses and cardiac chambers. Otherwise, the thickened left ventricle was a double-walled structure. The results of CMR also showed an abnormal structure in the myocardium. These results all confirmed the diagnosis of LVNC.

LVNC has not only a heterogeneous genetic background but also a heterogeneous pathogenetic background. ${ }^{17)}$ The ex-

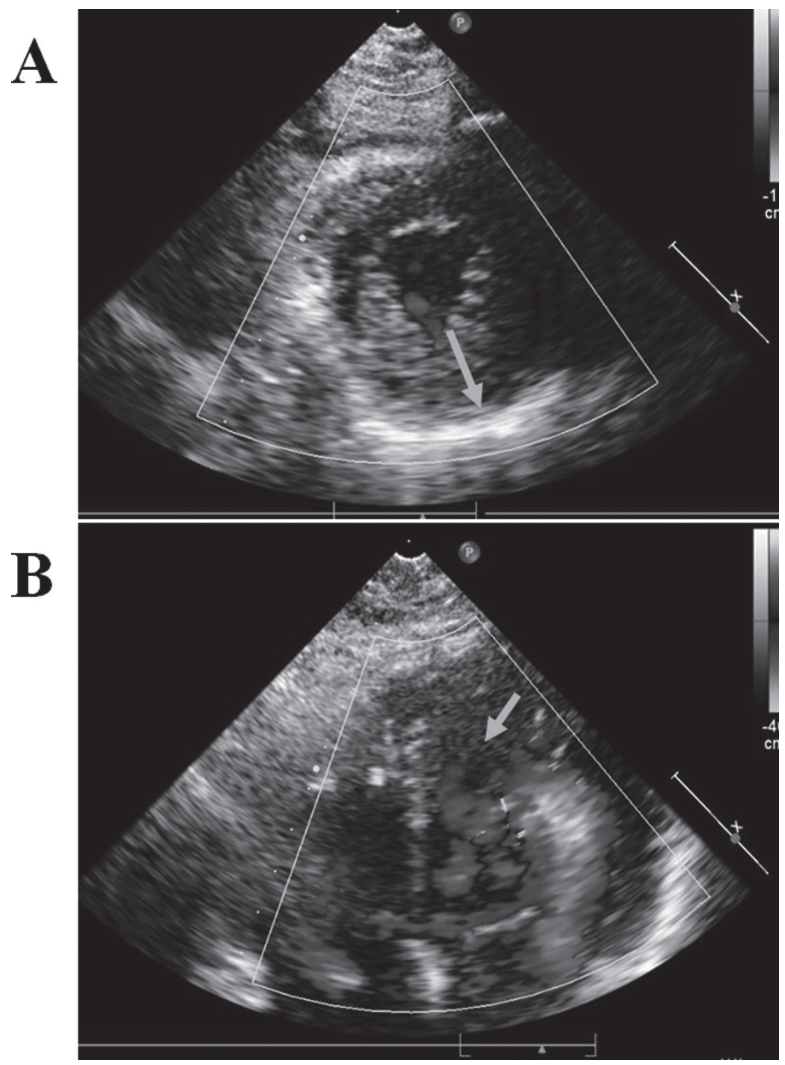

Figure 3. Echocardiography performed on April 12, 2012. Although the EF value increased significantly from $28 \%$ to $56 \%$, the echocardiogram showed the compacted and non-compaction layers were similar to those observed on the August 13, 2010 echocardiogram. A: short-axis view of heart, and B: 4-chamber view.

act cause of LVNC is unknown but it is believed that myocardial ischemia or pressure overload could be responsible for the development of the deep trabeculations. ${ }^{18)}$ In patients with LVNC, abnormal myocardial perfusion was common in both non-compacted and compacted myocardium. ${ }^{19)}$ The defective or dysfunctional myocardial microcirculation leads to myocardial ischemia and necrosis, which caused hypertrabeculation. ${ }^{17)}$ Another concept that may explain the pathogenesis of LVNC is that LVNC may develop secondarily by various mechanisms as an adaptive reaction of an insufficiently contracting myocardium. ${ }^{17)}$ For example, a frustrated attempt at hypertrophying the myocardium to compensate for myocardial impairment, which in turn induces the development of LVNC. ${ }^{20)}$

An excess of catecholamines in pheochromocytoma is usually accompanied by classical symptoms and signs, in particular paroxysmal, such as headache, sweating, palpitations, and hypertension. In some cases, severe cardiovascular complications (eg, myocardial infarction, heart failure) may occur. ${ }^{21)}$ In the current case, all the symptoms and complications could be observed. Furthermore, all the symptoms and complications were cured and there were no relapses even if the treatment was discontinued after the pheochromocytoma resection. These suggested that the signs of the patients were mainly induced by pheochromocytoma.

Increased levels of catecholamines lead to increased oxygen consumption, vasoconstriction, cardiac afterload, increased 
production of reactive oxygen species, and cell hypertrophy. ${ }^{22)}$ The myocardial ischemia, pressure overload, and hypertrophy, all of which resulted in the outbreak of HF, could have contributed to the progression of LVNC. Therefore, we speculated that in this patient, the LVNC was associated with the acute attack of HF, which was induced by the pheochromocytoma-secreted catecholamines. However, it could not be confirmed that the LVNC was caused by the pheochromocytoma in this patient because the LVNC was not improved after resection of the pheochromocytoma. The association of LVNC and the pheochromocytoma should be further investigated.

\section{REFERENCES}

1. Galetta F, Franzoni F, Bernini G, et al. Cardiovascular complications in patients with pheochromocytoma: a mini-review. Biomed Pharmacother 2010; 64: 505-9. (Review)

2. Stein PP, Black H. A simplified diagnostic approach to pheochromocytoma. Medicine 1990; 70: 46-66. (Review)

3. Lenders JW, Eisenhofer G, Mannelli M, Pacak K. Phaeochromocytoma. Lancet 2005; 366: 665-75. (Review)

4. Zelinka T, Petrák O, Turková H, et al. High incidence of cardiovascular complications in pheochromocytoma. Horm Metab Res 2012; 44: 379-84.

5. Müller G, Saint F, Hamy A, et al. Pheochromocytoma revealed by acute heart failure. When should we operate? Presented at the ESES Congress, Gothenburg May 25-26, 2012. Langenbecks Arch Surg 2013; 398: 729-33.

6. Udeoji DU, Philip KJ, Morrissey RP, Phan A, Schwarz ER. Left ventricular noncompaction cardiomyopathy: updated review. Ther Adv Cardiovasc Dis 2013; 7: 260-73. (Review)

7. Benjamin MM, Khetan RA, Kowal RC, Schussler JM. Diagnosis of left ventricular noncompaction by computed tomography. Proc (Bayl Univ Med Cent) 2012; 25: 354-6.

8. Engberding R, Bender F, Identification on of a rare congenital anomaly of the myocardium by two-dimensional echocardiography: persistence of isolated myocardial sinusoids. Am J Cardiol 1984; 53: 1733-4

9. Marathe SP, Vaideeswar P, Parikh R, et al. Noncompaction cardiomyopathy: manifestation as a surgical pitfall - rare but real. Asian
Cardiovasc Thorac Ann 2015; 23: 133-9.

10. Peters F, Khandheria BK, Botha F, et al. Clinical outcomes in patients with isolated left ventricular noncompaction and heart failure. J Card Fail 2014; 20: 709-15.

11. Tian T, Liu Y, Gao L, et al. Isolated left ventricular noncompaction: clinical profile and prognosis in 106 adult patients. Heart Vessels 2014; 29: 645-52.

12. Yuan L, Xie M, Cheng TO, et al. Left ventricular noncompaction associated with hypertrophic cardiomyopathy: echocardiographic diagnosis and genetic analysis of a new pedigree in China. Int $\mathrm{J}$ Cardiol 2014; 174: 249-59.

13. Chin TK, Perloff JK, Williams RG, Jue K, Mohrmann R. Isolated noncompaction of left ventricular myocardium. A study of eight cases. Circulation 1990; 82: 507-13.

14. Jenni R, Oechslin E, Schneider J, Attenhofer Jost C, Kaufmann PA. Echocardiographic and pathoanatomical characteristics of isolated left ventricular non-compaction: a step towards classification as a distinct cardiomyopathy. Heart 2001; 86: 666-71.

15. Stöllberger C, Finsterer J, Blazek G. Left ventricular hypertrabeculation/noncompaction and association with additional cardiac abnormalities and neuromuscular disorders. Am J Cardiol 2002; 90: 899-902.

16. Udeoji DU, Philip KJ, Morrissey RP, Phan A, Schwarz ER. Left ventricular noncompaction cardiomyopathy: updated review. Ther Adv Cardiovasc Dis 2013; 7: 260-73. (Review)

17. Finsterer J. Cardiogenetics, neurogenetics, and pathogenetics of left ventricular hypertrabeculation/noncompaction. Pediatr Cardiol 2009; 30: 659-81. (Review)

18. Rosa L, Salemi VM, Alexandre LM, Mady C. Noncompaction cardiomyopathy: a current view. Arq Bras Cardiol 2011; 197: e139. (Review, English, Portuguese, Spanish)

19. Gao XJ, Li Y, Kang LM, et al. Abnormalities of myocardial perfusion and glucose metabolism in patients with isolated left ventricular non-compaction. J Nucl Cardiol 2014; 21: 633-42.

20. Finsterer J, Stöllberger C, Schubert B. Acquired left ventricular noncompaction as a cardiac manifestation of neuromuscular disorders. Scand Cardiovasc J 2008; 42: 25-30.

21. Zelinka T1, Petrák O, Turková H, et al. High incidence of cardiovascular complications in pheochromocytoma. Horm Metab Res 2012; 44: 379-84.

22. Prejbisz A, Lenders JW, Eisenhofer G, Januszewicz A. Cardiovascular manifestations of phaeochromocytoma. J Hypertens 2011; 29: 2049-60. (Review) 